\title{
Epidermal growth factor receptor expression correlates with poor survival in gastric adenocarcinoma from Mexican patients: a multivariate analysis using a standardized immunohistochemical detection system
}

Armando Gamboa-Dominguez ${ }^{1}$, Claudia Dominguez-Fonseca ${ }^{1}$, Leticia QuintanillaMartinez $^{2}$, Edgardo Reyes-Gutierrez ${ }^{1}$, Dan Green ${ }^{3}$, Arturo Angeles-Angeles ${ }^{1}$, Raymonde Busch $^{4}$, Christine Hermannstädter ${ }^{5}$, Jörg Nährig ${ }^{5}$, Karl-Friedrich Becker ${ }^{2,5}$, Ingrid Becker ${ }^{5}$, Heinz Höfler ${ }^{2,5}$, Falko Fend ${ }^{5}$ and Birgit Luber ${ }^{5}$

${ }^{1}$ Department of Pathology, Instituto Nacional de Ciencias Medicas y Nutricion Salvador Zubiran, Mexico City, Mexico; ${ }^{2}$ GSF-Forschungszentrum für Umwelt und Gesundheit, Institut für Pathologie, Neuherberg, Germany; ${ }^{3}$ Department of Oncology, Instituto Nacional de Ciencias Medicas y Nutricion Salvador Zubiran, Mexico City, Mexico; ${ }^{4}$ Technische Universität München, Klinikum rechts der Isar, Institut für Medizinische Statistik und Epidemiologie, München, Germany and ${ }^{5}$ Technische Universität München, Klinikum rechts der Isar, Institut für Allgemeine Pathologie und Pathologische Anatomie, München, Germany

The aim of the study was to determine epidermal growth factor receptor (EGFR) expression in gastric adenocarcinoma by standardized immunohistochemistry and to correlate EGFR expression with clinical features and patient survival. EGFR expression was investigated in paraffin sections of resection specimens of 89 gastric carcinomas from Mexican Mestizo patients using standardized immunohistochemistry with antigen retrieval (Dako EGFRpharmDx ${ }^{\mathrm{TM}}$ assay detection system). Membrane staining of EGFR was evaluated in the neoplastic cells and graded using a semiquantitative score (0-3+). Of the 89 carcinomas examined, staining of neoplastic cells was weak in $17(19.1 \%$, score $1+)$, moderate in $16(18.0 \%$, score $2+)$, and strong in nine cases $(10.1 \%$, score $3+)$. EGFR reactivity was heterogeneous, frequently showing completely negative up to $3+$ positive areas within an individual tumor. EGFR reactivity score correlated with distant metastases $(P=0.002)$ and clinical stage $(P=0.033)$. EGFR score $0 / 1+$ was significantly associated with an increase in patient survival when compared to score $2+/ 3+(P=0.0006)$. In a multivariate analysis, EGFR positive cells in muscularis or subserosa $(P=0.004)$, distant metastases $(P=0.016)$ and residual disease $(P=0.039)$ were significantly correlated with decreased survival. The prognosis was associated with the EGFR reactivity score $(P=0.003)$, distant metastases $(P=0.0001)$ and residual disease $(P=0.012)$ in a univariate analysis. EGFR reactivity in neoplastic cells is an independent prognostic factor in gastric adenocarcinoma. The relevance of the heterogeneity in EGFR expression with regard to tumor progression, metastasis and anti-EGFR therapy needs to be studied.

Modern Pathology (2004) 17, 579-587, advance online publication, 9 April 2004; doi:10.1038/modpathol.3800085

Keywords: epidermal growth factor receptor (EGFR); EGFR reactivity score; anti-EGFR therapy

The importance of growth factors for the maintenance of tissue integrity as well as tissue remodelling and their implication in pathological disorders

Correspondence: Dr Birgit Luber, Trogerstr. 18, 81675 München, Germany.

E-mail: luber@lrz.tum.de

Received 20 June 2003; revised 13 November 2003; accepted 16 November 2003; published online 9 April 2004 is well established. EGFR (HER1) is a transmembrane protein that consists of an extracellular ligand binding domain, a transmembrane region and an intracellular domain with intrinsic tyrosine kinase activity. ${ }^{1}$ Ligand binding activates an intracellular signalling cascade that leads to the activation of the tyrosine kinase activity of EGFR. The EGFR family consists of four members, including HER1/erbB1, HER2/erbB2 or neu, HER3/erbB3 and HER4/erbB4. 
EGFR is expressed in many epithelial cell types and carcinomas derived thereof and in nonepithelial cells, for instance smooth muscle cells, fibroblasts and nerve cells.

EGFR is known as a central element for signal transduction and diversification. ${ }^{2}$ Of particular interest is the fact that overexpression of EGFR and EGFR-family members, like HER2, has been described in human cancers, in particular breast, bladder, colon, glioma, non-small-cell lung, pancreatic, ovarian, gastric, lung, salivary, head and neck tumors. ${ }^{2,3}$ Yasui et $a l^{4,5}$ have observed elevated levels of EGFR in advanced gastric carcinomas. Amplification of the EGFR gene has been described as a rare event in gastric carcinomas. ${ }^{6-8}$ Mutations in the EGFR gene have been detected in glioblastomas, breast, ovarian, prostate and lung carcinomas, ${ }^{9}$ but so far not in gastric carcinoma. Dysregulation of the EGFR signal transduction pathway is a clinical target for anticancer therapy. ${ }^{3}$ Various strategies have been used to inhibit EGFR activity, targeting both extracellular or intracellular receptor components. ${ }^{10,11}$

EGFR immunohistochemical reactivity has been investigated in colonic adenocarcinoma, and a scoring system evaluating EGFR reactivity has been proposed. ${ }^{12}$ EGFR positivity at the invasion front showed the strongest correlation with patient survival. ${ }^{12}$

The present study was undertaken to investigate EGFR reactivity in gastric carcinoma using an established detection system and a semiquantitative score, and to correlate it with clinical features and patient survival.

\section{Materials and methods}

\section{Patient selection}

Patients with a diagnosis of gastric adenocarcinoma who had undergone a total gastrectomy in the period from 1982 to 2001 in the Instituto Nacional de Ciencias Medicas y Nutricion Salvador Zubiran, with available clinical information and follow-up, were considered. CD-F. and DG collected all the clinical information and follow-up. Age at diagnosis, gender, survival time and cause of death were obtained from the charts. A blinded review of all the cases was made by two pathologists (AG-D. and IB) and a diagnosis according to Laurén's classification of gastric adenocarcinoma was rendered. ${ }^{13}$ Mexican Mestizo patients with available paraffin material and a morphologic diagnosis of poorly differentiated intestinal, mixed or diffuse-type adenocarcinoma in which UICC staging criteria ${ }^{14}$ could be applied, were included.

\section{EGFR immunohistochemistry}

A hematoxylin/eosin stained section was obtained for morphologic review and two consecutive sec- tions were mounted on charged slides for immunohistochemistry. Immunostainings for EGFR were performed using the Dako EGFRpharmDx ${ }^{\mathrm{TM}}$ assay detection system (Dako Corporation, Carpinteria, CA), which recognizes a $170 \mathrm{kDa}$ transmembrane receptor encoded by the human HER1 gene. The manual staining protocol was precisely followed, and no substitutions were made. After dewaxing in fresh xylene, $100 \%$ ethanol, $95 \%$ ethanol and $70 \%$ ethanol (four baths each), the slides were placed in a humid chamber for proteolytic digestion with proteinase K solution (100 $\mu$ l for $5 \mathrm{~min}$ ), and quenching of endogenous peroxidase for $5 \mathrm{~min}$. The primary antibody was incubated for $30 \mathrm{~min}$ followed by $30 \mathrm{~min}$ incubation with labelled polymer, and DAB localization of the positive cells. Counterstain was made with hematoxylin followed by 10 slide dips in a bath containing $37 \mathrm{mmol} / \mathrm{l}$ ammonia water. In every run control slides were included, which were provided to validate the performance of the reagents of the Dako EGFRpharmDx ${ }^{\mathrm{TM}}$ assay detection kit. The control slides contained sections of pelleted, formalin-fixed, paraffin-embedded cell line HT-29 with a moderate level of EGFR protein expression (positive control, IHC staining score of the cell pellet is $2.5 \pm 0.5$ ) and of the EGFR negative CAMA-1 cell line (negative control, score 0).

\section{EGFR Reactivity Evaluation}

Membrane staining was evaluated in the neoplastic cells and quantified and graded as recommended in the detection kit:

0 score

No staining observed, or membrane staining in $<10 \%$ neoplastic cells. Negative.

$1+$ score

Weak complete and/or incomplete membrane staining in $>10 \%$ neoplastic cells. Positive.

$2+$ score

Moderate complete and/or incomplete membrane staining in $>10 \%$ neoplastic cells. Positive.

$3+$ score

Strong complete and/or incomplete membrane staining in $>10 \%$ neoplastic cells. Positive.

Localization and intensity of reactivity was evaluated for mucosa, submucosa and deeper zones (muscle layer and subserosa). Evaluation was performed by two pathologists (AG-D. and FF) who were unaware of clinical features and survival. Statistical analyses were performed using Fisher's exact and $\chi^{2}$ tests when appropriate. Kaplan-Meier survival time analysis was used to correlate EGFR reactivity, localization of positive cells (surface or deep), pT, pN and $\mathrm{pM}$ and $\mathrm{R}$ status with clinical evolution. Differences in survival between subgroups were compared by log-rank test. Cox regression analysis was performed correlating EGFR reactivity, localization of positive cells, and stage 
with prognosis. A two-sided $P$-value less than 0.05 was considered to be statistically significant.

\section{Results}

\section{EGFR Score of Reactivity}

The clinicopathological features of 89 Mexican Mestizo patients with gastric cancer are shown in Table 1. The summarized results of EGFR immunohistochemistry are shown in Table 2. Of the 89 carcinomas examined, $47(52.8 \%)$ were negative or reactive in $<10 \%$ of neoplastic cells (score 0 ). Complete and/or incomplete membrane staining in $>10 \%$ of neoplastic cells was weak in $17(19.1 \%$, score $1+)$, moderate in $16(18.0 \%$, score $2+)$ and strong in nine cases $(10.1 \%$, score $3+$, Figure 1). The percentage of EGFR reactive cells per case was also evaluated, without considering the staining intensity. A total of 26 cases $(29.2 \%)$ were completely EGFR negative, 21 cases (23.6\%) showed reactivity in $<10 \%$ of neoplastic cells, 30 cases $(33.7 \%)$ were reactive in $10-50 \%$ of tumor cells and 12 cases $(13.5 \%)$ were positive in $>50 \%$ of neoplastic cells. Nerve and muscle cells served as reactive internal control. Normal gastric mucosa showed no EGFR staining. EGFR reactivity frequently showed a striking variability in the tumor tissue. In some cases, only a few tumor cells were highly reactive (score $3+$ ), while the rest of the tumor showed low reactivity or complete absence of EGFR expression (Figure 1B).

Table 1 Clinicopathologic features of 89 patients with gastric cancer

\begin{tabular}{lcr}
\hline Age & (Years) & \\
Mean & 57.8 & \\
Median & 60.0 & \\
Standard deviation & 15.2 & \\
Range & $14-86$ & \\
& $n$ & 49.4 \\
& & 50.6 \\
Gender & 44 & \\
Female & 45 & \\
Male & & 40.4 \\
& & 5.1 \\
Histotype (Laurén) & 36 & \\
Intestinal & 49 & 1.1 \\
Diffuse & 4 & 28.1 \\
Mixed & & 22.5 \\
Stage (UICC) & & 15.7 \\
IB & 1 & 32.6 \\
II & 25 & \\
IIIA & 20 & 22.5 \\
IIIB & 14 & \\
IV & 29 & \\
Residual disease & & \\
R0 & & \\
R1 & 69 & \\
& 20 & \\
\hline
\end{tabular}

Table 2 EGFR reactivity in 89 patients with gastric cancer

\begin{tabular}{lrc}
\hline & $n$ & $\%$ \\
\hline EGFR score of reactivity & & \\
0 & 47 & 52.8 \\
$1+$ & 17 & 19.1 \\
$2+$ & 16 & 18.0 \\
$3+$ & 9 & 10.1 \\
& & \\
Percentage of EGFR positive cells & \\
$0 \%$ & 26 & 29.2 \\
$<10 \%$ & 21 & 23.6 \\
$10-50 \%$ & 30 & 33.7 \\
$>50 \%$ & 12 & 13.5 \\
\hline
\end{tabular}

\section{EGFR Score and its Correlation with Clinicopathological Features and Morphology}

The EGFR score was correlated with clinicopathological features and morphology (Table 3). EGFR score significantly correlated with distant metastases and clinical stage, but not with histotype, perigastric lymph node status or residual disease.

\section{Influence of EGFR Score and Percentage of EGFR Reactive Neoplastic Cells on Survival}

The mean and median of the overall patient followup were 21.3. months or 12.0 months, respectively, with a range of 1-173 months and a standard deviation of 28.8 months. The Kaplan-Meier method was used to correlate EGFR score and percentage of reactive neoplastic cells with patient survival. The log-rank test indicates a global $P$-value of 0.0083 . When the percentage of EGFR reactive cells was correlated with patient survival, no EGFR reactivity or reactivity in $<10 \%$ cells resulted in increased patient survival when compared with EGFR reactivity in $10-50 \%$ or $>50 \%$ cells (Figure 3 ). This trend was observable, although the result did not reach statistical significance (log-rank test: global $P$-value 0.0688).

\section{Distribution of EGFR Reactive Neoplastic Cells and Association of EGFR Reactivity with Survival}

Localization and intensity of reactivity was evaluated for mucosa, submucosa and deeper zones (muscle layer and subserosa). The presence of EGFR reactive cells in the muscle layer or subserosa was significantly associated with a decrease in patient survival (log-rank test: global $P$-value 0.0004 , Figure 4).

A correlation between EGFR reactivity score, percentage and localization of positive cells, stage, distant metastases and residual disease status with prognosis was investigated by a univariate analysis (Table 4). EGFR reactivity score was associated with 


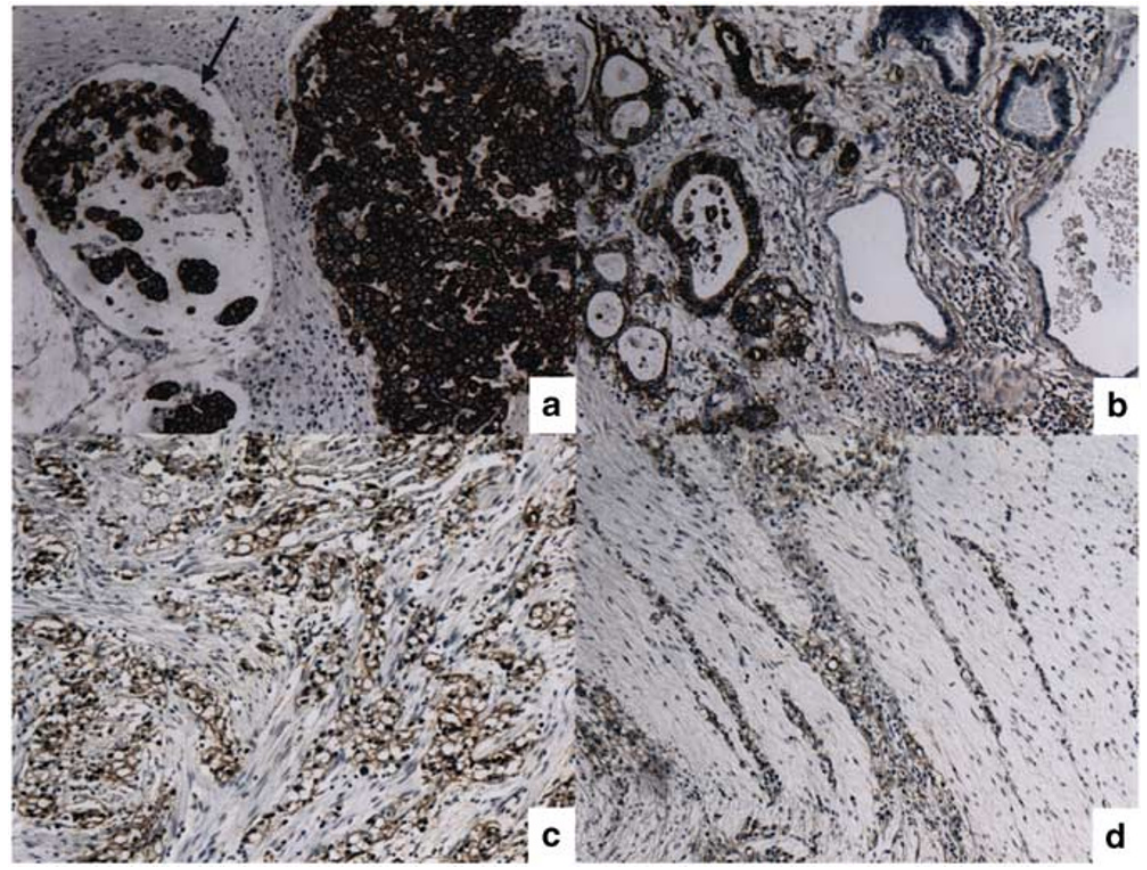

Figure 1 Immunohistochemical detection of EGFR in gastric carcinoma. Examples of different gastric adenocarcinomas displaying variable degrees of intensity of EGFR staining at the invasion front. (a) Adenocarcinoma of mixed type with $3+$ intensity of EGFR staining; the arrow indicates intralymphatic carcinoma. (b) Adenocarcinoma of intestinal type, example of heterogeneous staining with 0-3 + intensity. (c) Adenocarcinoma of diffuse type with $2+$ intensity of membrane staining. (d) Adenocarcinoma of diffuse type with $1+$ intensity of membrane staining. Original magnification: $\times 100$.

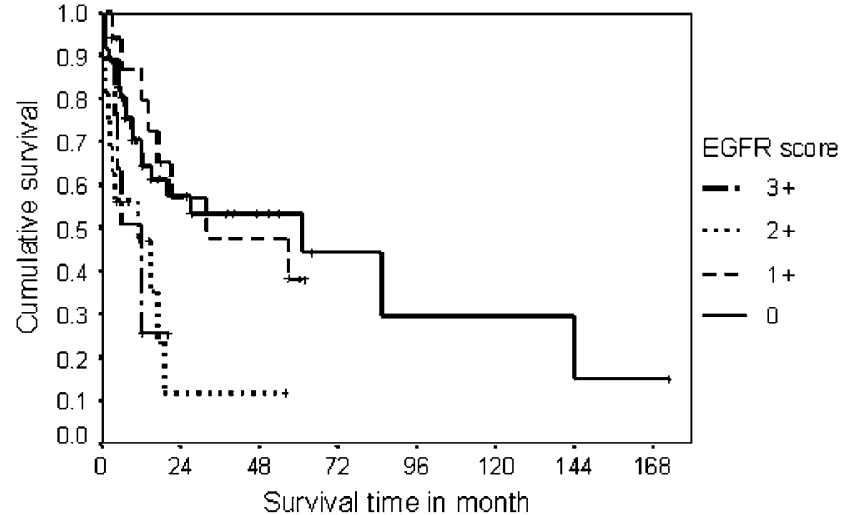

$\begin{array}{lll} & \text { log-rank } & \\ \text { Global } & 0.0083 & \text { s } \\ \text { Score 0 vs 1+ } & 0.9906 & \text { ns } \\ \text { Score 0 vs 2+ } & 0.0070 & \text { s } \\ \text { Score 0 vs 3+ } & 0.0552 & \text { ns } \\ \text { Score 1+ vs 2+ } & 0.0088 & \text { s } \\ \text { Score 1+ vs 3+ } & 0.0195 & \text { s } \\ \text { Score 2+ vs 3+ } & 0.8424 & \text { ns } \\ \text { Score 011+ vs 2+/3+ } & 0.0006 & \text { s }\end{array}$

Figure 2 Survival impact of EGFR score in gastric adenocarcinoma. The log-rank test statistical analysis indicates a global logrank $P=0.0083$ when the survival impact of EGFR score was evaluated. EGFR scores 0/1 + were significantly associated with increased survival when compared to $2+/ 3+(P=0.0006)$. s: significant; ns: not significant. the length of survival $(P=0.003)$. In contrast, the percentage of EGFR positive cells was not correlated with patient survival $(P=0.071)$, although a trend

Table 3 EGFR score of reactivity and its correlation with clinicopathological features and morphology in 89 Mexican Mestizo patients with gastric cancer

\begin{tabular}{|c|c|c|c|c|c|}
\hline & 0 & $1+$ & $2+$ & $3+$ & Total \\
\hline \multicolumn{6}{|c|}{ Histotype (Laurén) } \\
\hline Intestinal & 23 & 4 & 7 & 2 & 36 \\
\hline Mixed & 1 & 2 & 0 & 1 & 4 \\
\hline Diffuse & 23 & $\begin{array}{c}11 \\
P=0.201\end{array}$ & 9 & 6 & 49 \\
\hline \multicolumn{6}{|c|}{ Tumour invasion } \\
\hline pT2 & 7 & 1 & 0 & 0 & 8 \\
\hline pT3-4 & 40 & $\begin{array}{c}16 \\
P=0.304\end{array}$ & 16 & 9 & 81 \\
\hline \multicolumn{6}{|c|}{ Perigastric lymph node status } \\
\hline pNo & 13 & 5 & 1 & 2 & 21 \\
\hline $\mathrm{pN} 1-2$ & 34 & $\begin{array}{c}12 \\
P=0.313\end{array}$ & 15 & 7 & 68 \\
\hline \multicolumn{6}{|c|}{ Distant metastases } \\
\hline pM0 & 40 & 15 & 7 & 9 & 71 \\
\hline pM1 & 7 & $\begin{array}{c}2 \\
P=0.002\end{array}$ & 9 & 0 & 18 \\
\hline \multicolumn{6}{|c|}{ Residual disease } \\
\hline R0 & 39 & 13 & 10 & 7 & 69 \\
\hline R1 & 8 & $\begin{array}{c}4 \\
P=0.406\end{array}$ & 6 & 2 & 20 \\
\hline \multicolumn{6}{|c|}{ Clinical stage (UICC) } \\
\hline I-II & 19 & 5 & 1 & 1 & 26 \\
\hline III-IV & 28 & $\begin{array}{c}12 \\
P=0.033\end{array}$ & 15 & 8 & 63 \\
\hline
\end{tabular}




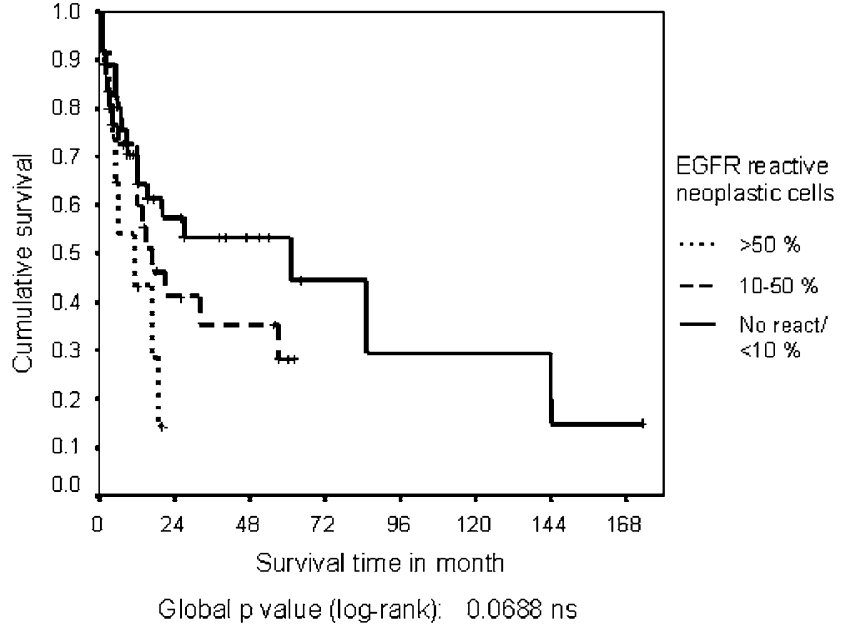

Figure 3 Influence of the percentage of EGFR reactive neoplastic cells on survival. The log-rank test statistical analysis indicates a $P=0.0688$ when the impact of the percentage of EGFR reactive neoplastic cells on survival was determined. ns: not significant.

was detectable. Furthermore, a signifcant association with survival was observed for positive neoplastic cells in muscularis or subserosa $(P=0.001)$, distant metastases $(P=0.0001)$ and residual disease $(P=0.012)$. A multivariate analysis using Cox's proportional hazard model revealed that EGFR positive cells in muscularis or subserosa $(P=0.004)$, distant metastases $(P=0.016)$ and residual disease $(P=0.039)$ were significantly correlated with a decrease in survival (Table 4). EGFR reactive tumor cells in muscularis or subserosa can therefore be considered as an independent prognostic factor.

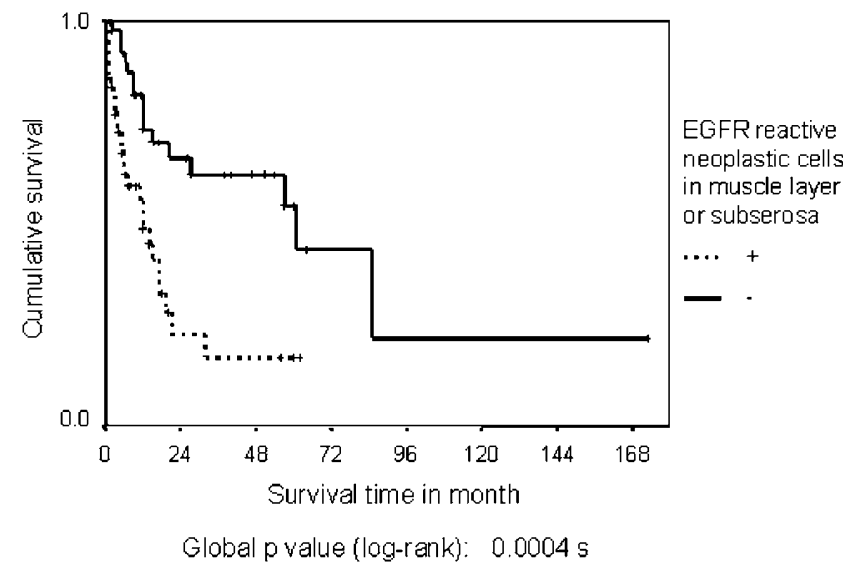

Figure 4 Influence of the presence of EGFR reactive cells infiltrating muscle layer and subserosa on survival. The log-rank test statistical analysis indicates a $P=0.0004$ when the impact of EGFR reactive cells infiltrating muscle layer or subserosa on survival was investigated. s: significant.

Kaplan-Meier survival time analysis was used to correlate residual disease status and EGFR score at the same time with patient survival. We decided to compare EGFR score $0 / 1+$ with EGFR score $2+/$ $3+$, since there was no significant difference in survival time in patients with EGFR score 0 and $1+$ (Figure 2). Survival time of patients with R0 resection with EGFR score 0/1 + was significantly increased when compared with patients with R0 resection and EGFR score $2+/ 3+$ or patients with R1 resection with or without EGFR reactivity (global $P$-value 0.0003 , Figure 5). A significant increase in survival time was also detected in patients without

Table 4 Analysis of prognostic factors in gastric carcinomas

\section{Univariate}

EGFR reactivity score

Percentage of EGFR reactive neoplastic cells

EGFR reactive cells in mucosa

EGFR reactive cells in submucosa

EGFR reactive cells in muscularis or subserosa

EGFR reactive cells in submucosa, muscularis or subserosa

Stage III-IV

Distant metastases

Residual disease
Significance (P-value)

0.003

0.071

0.019

0.124

0.001

0.002

0.064

0.0001

0.012

Multivariate: Cox proportional hazard model in stepwise forward fashion 95\% CI for relative risk

\begin{tabular}{|c|c|c|c|c|}
\hline & \multirow[b]{2}{*}{$\begin{array}{c}\text { Significance } \\
(P \text {-value })\end{array}$} & \multirow[b]{2}{*}{ Relative risk } & \\
\hline & & & Lower & Upper \\
\hline EGFR reactive cells in muscularis or subserosa & 0.004 & 2.679 & 1.373 & 5.224 \\
\hline Distant metastases & 0.016 & 2.583 & 1.190 & 5.607 \\
\hline Residual disease & 0.039 & 2.057 & 1.037 & 4.082 \\
\hline
\end{tabular}

CI, confidence interval. 
distant metastases and EGFR score $0 / 1+$ when compared with patients without distant metastases and EGFR score $2+/ 3+$ or patients with distant metastases with or without EGFR positive tumor cells (global $P$-value $<0.0001$, Figure 6). Finally, a significant decrease in survival time was detected in patients with stage III-IV and EGFR score $2+/ 3+$ when compared with patients in stage III-IV with EGFR score $0 / 1+$ or patients in stage I-II with or without EGFR positive tumor cells (global $P$-value 0.0015 , Figure 7).

A summary of the survival time analyses is shown in Table 5. Distant metastases combined with EGFR reactivity score (global $P$-value $<0.0001$ ), residual disease status combined with EGFR reactivity score (global $P$-value 0.0003 ), and EGFR reactive cells in muscularis or subserosa (global $P$-value 0.0004 ) revealed the strongest impact on survival.

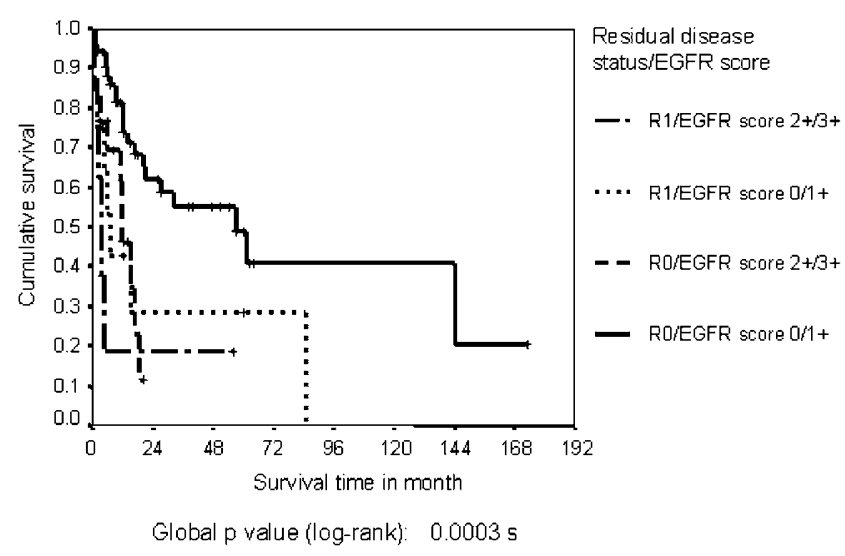

Figure 5 Influence of the residual disease status and EGFR score on survival. The log-rank test statistical analysis indicates a $P=0.0003$ when the impact of the residual disease status and EGFR score on survival was investigated. s: significant.

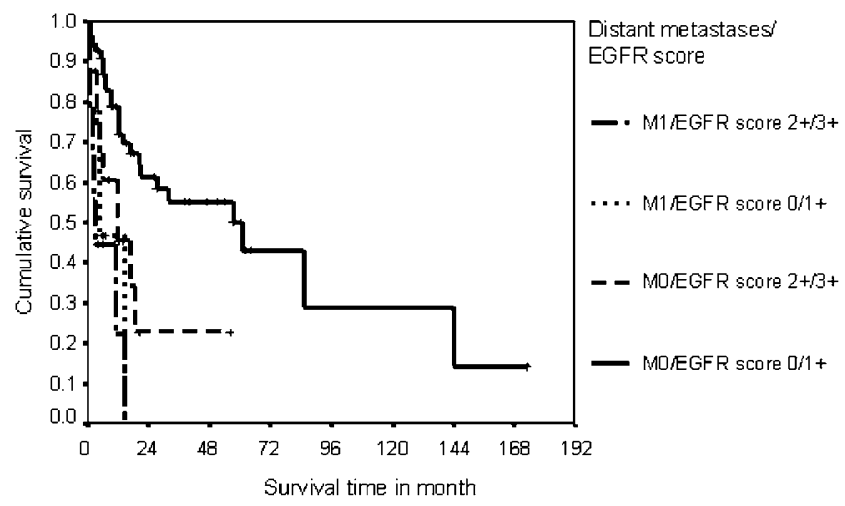

Global $p$ value (log-rank): $<0.0001 \mathrm{~s}$

Figure 6 Influence of the presence of distant metastases and EGFR score on survival. The log-rank test statistical analysis indicates a $P \leqslant 0.0001$ when the impact of the residual disease status on survival was investigated. s: significant.

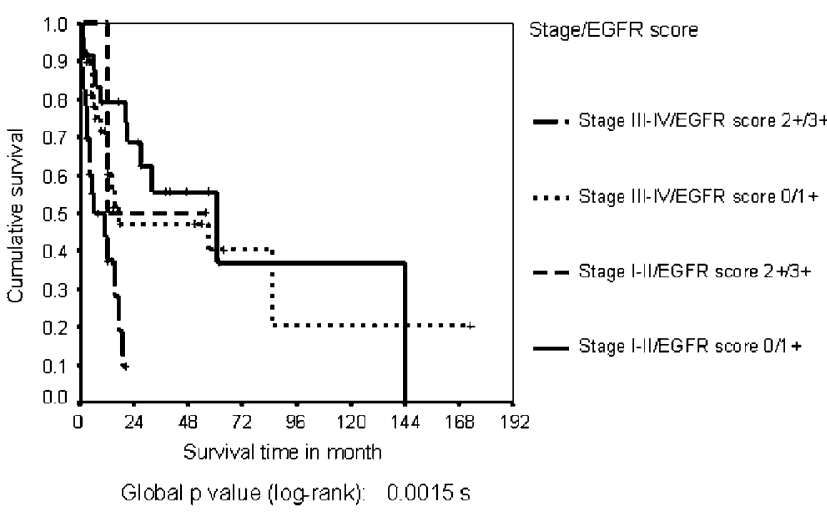

Figure 7 Influence of stage and EGFR score on survival, The logrank test statistical analysis indicates a $P=0.0015$ when the impact of stage on survival was investigated. s: significant.

Table 5 Summary of survival impacts

Global P-values (log-rank) in order of importance

\begin{tabular}{lc}
\hline $\begin{array}{l}\text { Distant metastases/EGFR } \\
\text { reactivity score }\end{array}$ & $<0.0001$ \\
$\begin{array}{l}\text { Residual disease status/EGFR } \\
\text { reactivity score }\end{array}$ & 0.0003 \\
$\begin{array}{l}\text { EGFR reactive cells in } \\
\text { mascularis or subserosa }\end{array}$ & 0.0004 \\
$\begin{array}{l}\text { Stage/EGFR reactivity score } \\
\text { EGFR reactivity score }\end{array}$ & 0.0015 \\
\end{tabular}

\section{Discussion}

EGFR and its ligands are frequently overexpressed in human cancers. $^{2,3}$ EGFR overexpression may result from gene amplification or mutation, transcriptional abnormalities or autocrine stimulation by enhanced expression of the ligands EGF and TGF $\alpha$. EGFR plays a critical role in tumor progression by stimulating cell cycle progression, invasion and metastasis. ${ }^{15}$ Specific abregation of EGFR results in cell cycle arrest, apoptosis or dedifferentiaton of cancer cells, which makes EGFR a promising target in cancer therapy. Available EGFR-targeted agents block either kinase activity or are monoclonal antibodies that bind the extracellular domain of the receptor, thereby competing with the natural ligands. Therapeutics against EGFR have entered clinical evaluation in malignant diseases. Clinical phase II and III studies with ZD1839 (Iressa), OSI774 (Tarceva) and Cetuximab (IMC-C225) have been started for several types of cancer, for instance colorectal carcinoma and non-small-cell lung carcinoma. ${ }^{16}$

Immunohistochemistry is the standard tool to determine EGFR overexpression and to identify patients who are most likely to benefit from therapy with anti-EGFR monoclonal antibody or kinase inhibitors. Although immunohistochemical 
analysis of EGFR expression in paraffin tissues is well established, there is a need to standardize the detection method and the scoring system. Otherwise, different positivity rates may be observed. This is especially critical for EGFR, since both percentage of positive cells as well as staining intensity are evaluated, similar to HER2 staining. In order to achieve the reproducibility and reliability required for a diagnostic test with potential impact on therapy, the Dako EGFRpharmDx ${ }^{\mathrm{TM}}$ assay detection system has been developed, and EGFR expression has already been investigated in colonic adenocarcinoma with this kit. ${ }^{12}$

In the present study, the Dako EGFRpharmDx ${ }^{\mathrm{TM}}$ assay has been used for the first time in gastric carcinoma. EGFR expression was observed in $47.2 \%$ of cases in the neoplastic cells, but not in normal gastric mucosa. In other studies, the observed positivity rates were in the range of 34$43 \% .{ }^{4,14,15,17}$ A significant association was found in the present study between EGFR reactivity score and distant metastases, clinical stage and patient survival. The presence of EGFR reactive cells in the muscle layer and subserosa was associated with a decrease in survival. Intratumoral EGFR expression was very heterogeneous, which indicates that EGFR overexpression might be a late event in tumor progression or might be related to genetic instability.

\section{EGFR as a Prognostic Marker in Gastric Cancer}

Our finding that EGFR reactivity score may serve as a prognostic indicator in gastric cancer is in accordance with several studies that have demonstrated that EGFR expression correlates with poor prognosis. ${ }^{18}$ Recently, the relationship between EGFR expression and cancer prognosis was investigated based on the analysis of literature data of more than 200 studies published between 1985 and $2000{ }^{18}$ It was found that EGFR expression was a strong prognostic indicator in cancers of the head and neck, ovary, cervix, bladder and esophagus, and that EGFR expression correlated with reduced recurrence-free and overall survival in $70 \%$ of studies included in the literature search. In gastric, breast, endometrial and colorectal cancers, EGFR expression was associated with poor survival in $52 \%$ of the included studies, while in non-small-cell lung cancer only $30 \%$ of studies showed such a correlation between EGFR expression and survival. The variations of the results between the different studies might be due to different methodical setups and further point to a need of a standardized EGFR immunohistochemistry. For gastric cancer, co-expression of EGFR and its ligands EGF or TGF- $\alpha$ was found to be correlated with a decrease of survival or the relapse-free survival interval. ${ }^{4,17,19}$ Amplification $^{7,8}$ or expression of EGFR ${ }^{4}$ was correlated with advanced clinical stage and the presence of lymph node metastasis. ${ }^{5,20}$

\section{EGFR Positivity in Muscle Layer or Subserosa}

In the present study, the presence and staining intensity of EGFR reactive cells were evaluated in mucosa, submucosa and at the deep invasion front in muscle layer and subserosa after exclusion of two patients with early cancer in muscosa and submucosa. The localization of EGFR reactive cells in muscle layer and subserosa was associated with a decrease in patient survival which indicates that EGFR positivity at the deep invasion front is critical in determining the patient's outcome. In a recent study using the same technique in colonic adenocarcinoma, positivity at the invasion front also showed the strongest correlation with survival duration as well as with EGFR positivity of lymph node and liver metastases. ${ }^{12}$ Increased EGFR expression at the most invasive parts of carcinomas has also been reported for oral squamous cell carcinomas. ${ }^{21}$ These data support the hypothesis that the invasive front of carcinomas is the most critical area for prognostication. ${ }^{12,22,23}$

\section{Heterogeneity of EGFR Expression}

EGFR reactivity showed a marked intratumoral heterogeneity, frequently showing a range of completely negative up to $3+$ positive neoplastic cells within an individual case. EGFR staining heterogeneity was also observed for colonic adenocarcinoma. ${ }^{12}$ These observations argue for an upregulation of EGFR expression in later stages of tumor progression. Different mechanisms, like autocrine stimulation by growth factors, genetic instability or transcriptional deregulation, may be considered. Amplification of the EGFR gene has been described in gastric carcinomas, ${ }^{6-8}$ but no mutations have been found so far. ${ }^{9}$ With regard to anti-EGFR therapy, the impact of EGFR heterogeneity on the therapeutic response has to be clarified. It may also be of importance in the evaluation of small tumor samples, for example, pretherapeutic endoscopic samples.

\section{Geographic Influence}

Our study is the first study that investigates EGFR in tumor samples of Mexican gastric carcinoma patients while other studies frequently investigated samples of Japanese, ${ }^{4,5,7,8,17,19,20}$ Northern Ameri$\mathrm{can}^{24}$ or European ${ }^{25,26-30}$ gastric carcinoma patients. Although Mexico is a country with a higher incidence of gastric cancer compared to most Western countries, the distribution of histologic subtypes is similar. ${ }^{31}$ Overall, our study of Mexican patients produced similar results in terms of 
incidence and prognostic influence of EGFR expression and lack of correlation with histologic features as studies with other ethnic groups, ${ }^{25}$ suggesting that the ethnic and geographic origin of patients is unlikely to play a role in EGFR expression.

Tumor progression to an invasive state includes up-regulation of cellular motility, and EGFR is frequently involved in this process, ${ }^{32,33}$ Anti-EGFRbased therapies aim to interfere with tumor invasion and metastasis. A standardized EGFR scoring system is a prerequiste to define patient subgroups who are likely to benefit from anti-EGFR therapy.

\section{Acknowledgements}

Dr Armando Gamboa-Dominguez obtained an award of the International Union Against Cancer (UICC ICRETT 552). This work has been partially supported by a UICC International Cancer Technology Transfer Fellowship and by a grant to Drs B. Luber, $\mathrm{KF}$. Becker and H. Höfler from the Deutsche Forschungsgemeinschaft (SFB 456). The authors thank E. Samson, J. Müller, S. Roth and N. Kink for excellent technical assistance. Neither author has a financial or other relation with Dako Corporation. Neither author received financial support from Dako Corporation or has a conflict of interest. The epidermal growth factor detection system was purchased from Dako Corporation, who did not influence the results of this study.

\section{References}

1 Riese DJ, Stern DF. Specificity within the EGF family/ ErbB receptor family signaling network. Bioessays 1998;20:41-48.

2 Prenzel N, Zwick E, Leserer M, et al. Tyrosine kinase signalling in breast cancer. Epidermal growth factor receptor: convergence point for signal integration and diversification. Breast Cancer Res 2000;2:184-190.

3 Hong WK, Ullrich A. The role of EGFR in solid tumors and implications for therapy. Oncol. Biother 2000;1: 2-29.

4 Yasui W, Hata J, Yokozaki H, et al. Interaction between epidermal growth factor and its receptor in progression of human gastric carcinoma. Int J Cancer 1988;41: 211-217.

5 Yasui W, Sumiyoshi H, Hata J, et al. Expression of epidermal growth factor receptor in human gastric and colonic carcinomas. Cancer Res 1988;48:137-141.

6 Lemoine NR, Jain S, Silvestre F, et al. Amplification and overexpression of the EGF receptor and c-erbB-2 proto-oncogenes in human stomach cancer. Br J Cancer 1991;64:79-83.

7 Tsugawa K, Yonemura Y, Hirono Y, et al. Amplification of the c-met, c-erbB-2 and epidermal growth factor receptor gene in human gastric cancers: correlation to clinical features. Oncology 1998;55:475-481.

8 Hirono Y, Tsugawa K, Fushida S, et al. Amplification of epidermal growth factor receptor gene and its relationship to survival in human gastric cancer. Oncology 1995;52:182-188.

9 Kuan CT, Wikstrand CJ, Bigner DD. EGF mutant receptor vIII as a molecular target in cancer therapy. Endocr Relat Cancer 2001;8:83-96.

10 Zwick E, Bange J, Ullrich A. Receptor tyrosine kinase signalling as a target for cancer intervention strategies. Endocr Relat Cancer 2001;8:161-173.

11 Mendelsohn J. The epidermal growth factor receptor as a target for cancer therapy. Endocr Relat Cancer 2001;8:3-9.

12 Goldstein NS, Armin M. Epidermal growth factor receptor immunohistochemical reactivity in patients with American Joint Committee on Cancer Stage IV colon adenocarcinoma: implications for a standardized scoring system. Cancer 2001;92:1331-1346.

13 Laurén P. The two histological main types of gastric carcinoma: diffuse and so-called intestinal-type of carcinoma. Acta Pathol Microbiol Scand 1965;64:31-49.

14 Spiessl B, Beahrs OH, Hermanek P, et al. editors. UICC International Union Against Cancer: TNM Atlas. Berlin, Heidelberg, Springer, 1992.

15 Lui VW, Grandis JR. EGFR-mediated cell cycle regulation. Anticancer Res 2002;22(1A):1-11.

16 de Bono JS, Rowinsky EK. The ErbB receptor family: a therapeutic target for cancer. Trends Mol Med 2002;8(4 Suppl):S19-26.

17 Yonemura Y, Takamura H, Ninomiya I, et al. Interrelationship between transforming growth factor-alpha and epidermal growth factor receptor in advanced gastric cancer. Oncology 1992;49:157-161.

18 Nicholson RI, Gee JM, Harper ME. EGFR and cancer prognosis. Eur J Cancer 2001;37(Suppl 4):S9-S15.

19 Tokunaga A, Onda M, Okuda T, et al. Clinical significance of epidermal growth factor (EGF), EGF receptor, and c-erbB-2 in human gastric cancer. Cancer 1995;75(Suppl 6):1418-1425.

20 Iida A, Hirose K, Arai M, et al. Relationships among the expression of epidermal growth factor receptor, proliferating cell nuclear antigen labeling index, and lymph node metastasis in gastric cancer. Oncology 1995;52:189-195.

21 Bankfalvi A, Krassort M, Buchwalow IB, et al. Gains and losses of adhesion molecules (CD44, E-cadherin, and beta-catenin) during oral carcinogenesis and tumour progression. J Pathol 2002;198:343-351.

22 Bryne M, Boysen M, Alfsen CG, et al. The invasive front of carcinomas. The most important area for tumour prognosis? Anticancer Res 1998;18(6B): 4757-4764.

23 Bankfalvi A, Piffko J. Prognostic and predictive factors in oral cancer: the role of the invasive tumour front. J Oral Pathol Med 2002;29:291-298.

24 Lee EY, Cibull ML, Strodel WE, et al. Expression of HER-2/neu oncoprotein and epidermal growth factor receptor and prognosis in gastric carcinoma. Arch Pathol Lab Med 1994;118:235-239.

25 Koullias GJ, Kouraklis GP, Raftopoulos IS, et al. Increased estrogen receptor and epidermal growth factor receptor gene product co-expression in surgically resected gastric adenocarcinomas. J Surg Oncol 1996;63:166-171.

26 Sanz-Ortega J, Steinberg SM, Moro E, et al. Comparative study of tumor angiogenesis and immunohistochemistry for p53, c-ErbB2, c-myc and EGFr as prognostic factors in gastric cancer. Histol Histopathol 2000;15:455-462. 
27 Slesak B, Harlozinska A, Porebska I, et al. Expression of epidermal growth factor receptor family proteins (EGFR, c-erbB-2 and c-erbB-3) in gastric cancer and chronic gastritis. Anticancer Res 1998;18: 2727-2732.

28 D’Agnano I, D’Angelo C, Savarese A, et al. DNA ploidy, proliferative index, and epidermal growth factor receptor: expression and prognosis in patients with gastric cancers. Lab Invest 1995;72:432-438.

29 Filipe MI, Osborn M, Linehan J, et al. Expression of transforming growth factor alpha, epidermal growth factor receptor and epidermal growth factor in precursor lesions to gastric carcinoma. $\mathrm{Br} \mathrm{J}$ Cancer 1995;71:30-36.
30 Karameris A, Kanavaros P, Aninos D, et al. Expression of epidermal growth factor (EGF) and epidermal growth factor receptor (EGFR) in gastric and colorectal carcinomas. An immunohistological study of 63 cases. Pathol Res Pract 1993;189:133-137.

31 Mohar A, Suchil-Bernal L, Hernandez-Guerrero A, et al. Intestinal type: diffuse type ratio of gastric carcinoma in a Mexican population. J Exp Clin Cancer Res 1997;16:189-194.

32 Liotta LA, Kohn EC. The microenvironment of the tumour-host interface. Nature 2001;411:375-379.

33 Wells A, Kassis J, Solava J, et al. Growth factor-induced cell motility in tumor invasion. Acta Oncol 2002;41:124-130. 